Techno-species: the bumanity that makes itself and the disposable

\title{
Tecno-especies: la bumanidad que se hace a si misma y los desechables
}

Mateja Kovacic

University of Oxford

María G. Navarro

Universidad de Salamanca

DOI: https://doi.org/10.15366/bp2021.27.002

Bajo Palabra. II Época. No 27. Pgs: 45-62

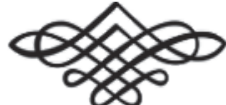


Recibido: 18/10/2020

Aprobado: 07/05/2021

\section{Resumen}

La cultura popular sigue alimentando el imaginario colectivo con cosas, humanas y no humanas, en las que podríamos convertirnos o con las que podríamos vernos confrontados. Además de robots, otras figuras significativas de la ficción popular que generaron imágenes son los seres humanos no humanos y los ciborgs, incorporadas a realidades socioculturales históricamente diversas. Los robots y la inteligencia artificial están redefiniendo el orden natural y su estructura jerárquica. No es raro, pues el orden natural siempre fluye, moldeado por los nuevos descubrimientos científicos, en especial la lectura del código genético, que revela y redefine las relaciones entre las formas de vida. Sin embargo, por primera vez, se está introduciendo una nueva especie artificial en los esquemas existentes y, por primera vez, parece hibridar el orden mundial natural antropocéntrico.

Palabras clave: Inteligencia artificial, Robots sociales, Aprendizaje automático, Posthumanismo, Tecnopersonas

\section{Abstract}

Popular culture continues fuelling public imagination with things, human and non-human, that we might become or confront. Besides robots, other significant tropes in popular fiction that generated images include non-human humans and cyborgs, wired into historically varying sociocultural realities. Robots and artificial intelligence are redefining the natural order and its hierarchical structure. This is not surprising, as natural order is always in flux, shaped by new scientific discoveries, especially the reading of the genetic code, that reveal and redefine relationships between life forms. However, for the first time, a new, artificial, species is being introduced into the existing diagrams, and, for the first time, it appears to hybridise the anthropocentric natural world order.

Keywords: Artificial Intelligence; Social Robots; Machine Learning; Posthumanism; Tecnopersons 


\section{De Humano híbrido a Robot híbrido ${ }^{1}$}

LOS ACADÉMICOS QUE ESCRIBEN sobre posthumanismo y tecno-humanidad suelen empezar aludiendo a la pionera evaluación de la nueva especie humana híbrida de Donna Haraway (1991) y Katherine Hayles (2004 y 2006); una mezcla de especie no individual, orgánica e inorgánica, sin género y no binaria. Rosi Braidotti (2006) y Katherine Hayles (2004 y 2006) plantearon que vivimos en la era posthumana, definida por una multitud de formas en las que la tecnología remodela y redefine los seres humanos y el lugar que ocupan en el universo antropocéntrico y antropogénico. Al mismo tiempo, la cultura popular sigue alimentando el imaginario colectivo con cosas, humanas y no humanas, en las que podríamos convertirnos o con las que podríamos vernos confrontados. Además de robots, otras figuras significativas de la ficción popular que generaron imágenes son los seres humanos no humanos y los ciborg, incorporadas a realidades socioculturales históricamente diversas. Tatsumi Takayuki, por ejemplo, presentó en su libro Full Metal Apache: Transactions Between Cyberpunk Japan and Avant-pop America (2006) la figura popular de posguerra del «full metal Apache» [«el apache metálico»]: un ser no japonés metalívoro en la cultura metalocéntrica. Esta imaginería metalocéntrica apareció tras la Segunda Guerra Mundial y, vinculada a la revolución industrial, rendía homenaje a las innovaciones y la proliferación de la industria siderúrgica que alimentaba las economías y los ejércitos de las naciones industrializadas (Kovacic 2016, 139). Dicho de otro modo, el ser humano de hierro apuntaba a un nuevo modo de existencia, cada vez más dependiente de la producción industrial.

Antes del ser humano de hierro estaba el monstruo de Shelley. En una novela de 1818, Frankenstein, Shelley nos ofrece un relato sobre una criatura ensamblada con distintas partes corporales de cadáveres humanos y dotada de vida por un científico, el doctor Frankenstein. A diferencia del ciborg moderno, esta criatura no había sido creada con intenciones transhumanistas, sino que más bien era un producto de las ciencias aplicadas que cada vez más interiorizaban el poder de la creación. Por insólito que pueda resultar, pues el monstruo era un ensamblaje de cadáveres, se trataba

\footnotetext{
${ }^{1}$ Este trabajo forma parte de los desarrollos de dos proyectos «El desván de la razón: cultivo de las pasiones, identidades éticas y sociedades digitales» (PAIDESOC) [FFI2017-82535-P] y «Democracia deliberativa y capital social en contextos interculturales» [CAS18/00351] este último desarrollado en University of Oxford.
} 
de una forma de vida orgánica sin ninguna intervención mecánica en su cuerpo, ni en su mente. El monstruo de Frankenstein sigue siendo una referencia corriente en la opinión pública cuando se discute sobre las intervenciones tecnológicas y biotecnológicas en la vida orgánica: seres humanos, plantas y mamíferos en particular. Shelley escribió su novela en plena revolución industrial de la era victoriana, cuando los científicos buscaban eslabones perdidos en la Gran Cadena del Ser; una época en la que se clasificaban e investigaban como parte del mundo natural «especies» tan extraordinarias como las sirenas, que ofrecían una explicación potencial de la evolución de la vida marina hasta convertirse en humana. Beatrice Laurent, que escribió sobre las sirenas diciendo que eran "eslabones perdidos», señalaba que en la época victoriana había tres teorías principales que moldeaban el discurso sobre las sirenas: la teoría creacionista que creía en un orden natural fijado desde la eternidad, la teoría evolutiva que consideraba que las sirenas eran un eslabón perdido entre las formas de vida marinas y terrestres y una tercera perspectiva, que explicaba que las sirenas eran antepasados comunes de diversas especies (Laurent 2018, 2). Como no podía ser de otra manera, este proceso tenía lugar en el siglo XIX; justo antes de que en 1859 Darwin publicara su teoría de la evolución en El origen de las especies y en el momento culminante de la expansión colonial que abrió el mundo, con todas sus maravillas a la investigación científica sobre los orígenes de la vida y los vínculos entre las especies dispersas por todo el planeta.

La especie popular en las décadas de 1980 y 1990, el «ciborg» (el término que fue acuñado por Manfred Clynes y Nathan Kline en 1960), era de manera similar un producto de la reconceptualización darwinista; en esta ocasión, la evolución artificial. Esto imprimía un giro poshumano y transhumano a la evolución humana cuando, por primera vez, el ser humano se convierte en una tecno-especie, aunque el homo sapiens, como especie, siempre ha dependido de la tecnología creada para compensar la falta de especialización que podemos encontrar en otros animales (Gehlen, 1988). Ahora mismo está produciéndose otro desplazamiento evolutivo con la promesa de formas de vida artificiales absolutamente nuevas: no híbridos de lo orgánico y lo inorgánico, sino híbridos de lo digital y lo físico.

Por consiguiente, la especie humana híbrida no se circunscribe a nuestra experiencia contemporánea: la búsqueda de ella y las tentativas de crearla han moldeado los paradigmas científicos desde hace siglos. Un ejemplo son los robots mecánicos (autómatas) —aparecidos por primera vez en Europa en el siglo XV tras la invención del mecanismo del reloj-, que emulaban la vida humana y animal en un intento de intimidar e impresionar. Pero tenían una finalidad que trascendía la reproducción fenomenológica de la vida: su existencia estaba entrelazada con negociaciones con la ontología de la vida en el orden natural. Muchos siglos más tarde, 
en 1920, los robots de Karel Capek (quien introdujo y popularizó la palabra «robot») pasaron a formar parte del cuestionamiento del orden social como metáfora marxista de la explotación de la mano de obra humana (1920/2001). Basándose en ellos, la nueva tecno-especie no solo lleva en fase de construcción desde hace siglos, sino que también ha moldeado la comprensión y la organización de los órdenes social, natural y artificial y ha impactado en la forma en que la filosofía natural y la ciencia definieron la vida y cómo han tratado de intervenir en ella.

Las primeras máquinas capaces de moverse de forma automática aparecieron en la antigua China $^{2}$ y la antigua Grecia y las movían el aire, el agua y la arena. Estaban automatizadas para realizar determinadas actividades, pero siglos después, el mecanismo del reloj permitió que estas máquinas exhibieran movimientos más parecidos a la vida y realizaran acciones más complejas. Los primeros autómatas de relojería, desde el pato mecánico que hacía la digestión de Jacques de Vaucanson (1739) hasta el célebre clarinetista de Cornelis Jacobus van Oeckelen (1838), también eran percibidos como proto-robots; una muestra de que los robots no vienen impulsados exclusivamente por la investigación de principios científicos, ni por el deseo de sustituir el trabajo humano y ganar eficiencia y velocidad y reducir costes — valores, todos ellos, de la economía capitalista-, sino que parte de la indagación para construir robots era el deseo de crear una forma de vida, una nueva especie.

El monstruo de Shelley, el autómata mecánico y los robots sociales contemporáneos reflejan la época antropogénica durante la que fueron y han sido creados. Norbert Wiener, autor del manifiesto de 1948 Cybernetics: Or Control and Communication in the Animal and the Machine, dividía la historia de los autómatas en cuatro etapas: la era golémica mítica, la edad de los relojes de los siglos XVII y XVIII, la era del vapor y la era del control y las comunicaciones (38-44). De acuerdo con ello, Wiener señalaba que estas fases generaron cuatro modelos del cuerpo humano: el cuerpo como figura de barro mágica, el cuerpo como mecanismo de relojería, el cuerpo como máquina de vapor y el cuerpo como sistema electrónico (citado en Tomas 1995, 23). Con las interpretaciones y las percepciones del cuerpo llegaron modelos externos que los emularon. Desde Descartes, que pensaba que los seres humanos eran autómatas según su filosofía mecanicista (1972) y la totalidad de la conceptualización moderna del universo como mecanismo de relojería, hasta los roboticistas de la época que construyen y estudian robots para comprender la psicología humana (Menzel y D'Aluisio, 2000) y que recurren a estudios sobre ni-

\footnotetext{
${ }^{2}$ Se pueden encontrar referencias a máquinas automáticas en China en los siglos X y XI a. C. y en Grecia en el siglo I.
} 
ños para culturizar robots, los seres humanos y los mecanismos automatizados han atravesado una historia intercambiable y entrelazada.

Los robots sociales contemporáneos — robots que coexisten con seres humanos en entornos sociales y alcanzan diversos grados de interacción social- ya no son meros modelos. Están convirtiéndose en una nueva especie. Esto supone una reconsideración continua de la relación entre el ser humano y la máquina que acabará cada vez más definida en términos de relaciones interespecíficas. Esta no es una cuestión del asunto mente-cuerpo digital (Katherine Hayles, «Flesh and Metal: Reconfiguring the Mindbody in Virtual Environments» [2006, 11]), sino de trascender de las formas más extremadamente inesperadas los límites existentes entre el mundo virtual y físico; no mediante extensiones humanas hacia lo virtual, sino mediante la extensión digital hacia el mundo de los robots y, por tanto, dando al mundo virtual el cuerpo y, con ello, la capacidad de manipular el entorno físico. Contemplar cómo se despliega este fenómeno —el crecimiento de la autonomía, la inteligencia y la independencia robótica - evoca la cuestión del fantasma de la máquina (Koestler, 1967): la conciencia nacida en el seno del cuerpo mecánico y el cerebro electrónico. Katherine Hayles ha sostenido que la metáfora del ciborg es obsoleta y ha afirmado que el ciborg ya no es una metáfora adecuada para explicar la condición humana contemporánea porque la cognosfera ha sustituido al ciborg y, a diferencia del ciborg, la cognosfera no está limitada a lo binario; más bien, es un «símbolo y una ejemplificación de los flujos cognitivos dinámicos existentes entre lo humano, lo animal y la máquina [...] La cognosfera, como el propio mundo, no es binaria, sino múltiple; no es una criatura dividida sino un complejo sistema con unas conexiones muy tupidas que evolucionan en compañía» (Hayles, «Unfinished Work» 165). Aunque lo digital se ha convertido de hecho en la nueva naturaleza humana, ya no define en exclusiva la naturaleza humana: para una multitud de robots sociales, desde robots humanoides de servicio hasta vehículos autónomos, se ha convertido en un paso evolutivo: en el origen de una nueva especie.

Con diversas manifestaciones del mundo digital en el mundo físico a través de los robots, la pregunta deja de ser si es posible una nueva especie, sino qué suponen estas posibilidades tecnológicas en la reestructuración de la arquitectura del universo antropogénico. La inteligencia artificial y los robots evolucionan juntos en relación simbiótica. Los valiosos conceptos de ciborg y del monstruo de Frankenstein nos recuerdan que el robot social es un ensamblaje. El robot comporta movilidad, manipulación y percepción controlada por una multitud de elementos de software desarrollados de forma independiente, pero que operan de forma interdependiente (The Computing Community Consortium Workshop 2009, 59). Dicho de otro modo: el robot es al mismo tiempo un ser físico y de información, con sus circuitos 
electrónicos enchufados constantemente a la red de trabajo. Como tales, los robots se dedican a la logística, el transporte, la atención sanitaria, el hogar, la educación y las plataformas sociales porque integran y hacen posible la automatización de servicios y son capaces de ampliar físicamente las plataformas de intercambio de datos hacia el mundo físico. Al mismo tiempo, la sociedad humana sigue definiéndose a través de su papel y sus necesidades en las redes socio-técnicas. Por ejemplo, el reciente programa nacional del gobierno de Japón, Society 5.0 o Super Smart Society, concibe una coexistencia ecosistémica de seres humanos y robots: desde vehículos autónomos, robots para la atención sanitaria telemática o robots sociales humanoides hasta drones y ayudantes personales de inteligencia artificial (MEXT 2016). Si los robots son la nueva especie, entonces el Internet de Todo, el despliegue total del mundo conectado, es la nueva arquitectura mundial. Esto requiere una evaluación de si los seres humanos se están volviendo a sí mismos obsoletos en el nuevo orden tecno-natural que se está creando con cada integración adicional de las tecnologías robóticas y de inteligencia artificial en la vida cotidiana. Mientras que «seres humanos obsoletos» se refiere en su mayoría a la capacidad de trabajo y de adquirir destrezas, los gobiernos del mundo están profesando la necesidad de rejuvenecerse robóticamente y mejorar a su población debido a la decreciente tasa de natalidad y al creciente número de personas ancianas y dependientes. En otras palabras, si el autómata de relojería reflejaba el deseo de crear formas de vida, las agendas nacionales de robótica actuales reflejan la necesidad de ellos. Los robots sociales, muchos de los cuales están todavía en fase experimental, están infiltrándose en la vida cotidiana como parte de la sociedad humana e hibridando sus rutinas diarias.

En su teorización visual de la evolución de los robots hasta convertirse en una nueva especie, Menzel y D’Aluisio (2000) denominaron Robo sapiens a esta última. La evolución del robot está limitada por el desarrollo de la inteligencia artificial y, por lo tanto, representa una nueva forma de encarnación inteligente, comparable al Homo sapiens. En un giro sobre la especie del Robo sapiens, Jennifer Robertson, una antropóloga que escribía sobre Japón, introdujo en su libro sobre los robots en Japón una nueva clasificación en el seno de la especie: Robo sapiens Japanicus (2018). Esta terminología, que refleja un sistema clasificatorio de la taxonomía de Linneo, tiene un valor simbólico cuando es ampliada por los antropólogos para que incluya formas de vida artificial. En 1735, Carl Linneo introdujo en su breve obra Systema Naturae un sistema para clasificar los seres vivos y sentó las bases del moderno sistema de clasificación. Propuso una estructura jerárquica de denominación de animales y plantas que transformó el modo en que se comprendía, percibía e investigaba el mundo natural. Incluir a los robots en la misma estructura organizativa comporta un nuevo tipo de orden tecno-natural. La obra de Robertson 
llega aún más lejos: en lugar de suponer la existencia de robots como una especie universal y singular, plantea la multiplicidad de especies de robots introduciendo la familia (Japanicus) además de las categorías taxonómicas de la especie (Robo) y el género (Sapiens). El sistema de clasificación de Linneo ha venido experimentando una transformación revolucionaria con el desarrollo de las tecnologías del ADN: los seres vivos y las relaciones que mantienen están siendo redefinidas por el código genético, en lugar de por la morfología. Sin embargo, mediante el «descubrimiento» de una nueva especie de robot en Japón, Robertson no está dando a entender que los robots hayan alcanzado un nivel de desarrollo por el que se pueda afirmar que son una nueva forma de vida. Más bien, considera que los robots son una tecnología inextricablemente vinculada a las estructuras sociales de Japón y moldeadas por las prácticas culturales de especificidad local. Curiosamente, no obstante, es precisamente esta diversidad cultural y este vínculo societario lo que define a cada nueva especie. Históricamente, la ciencia moderna y el lugar de lo humano en el orden mundial natural han sido afirmados no tras homogeneizar la vida sobre la tierra, sino al descubrir lo heterogénea que era, lo atada que estaba a los versátiles ecosistemas hallados en la Tierra.

En su obra El origen de las especies, Charles Darwin empleó una metáfora del árbol de la vida como teórica representación jerárquica de la totalidad de la vida sobre la Tierra y explicación de su teoría de la evolución y la interdependencia de las relaciones entre especies. En versiones posteriores, incluida la de Ernst Haeckel (18341919), el árbol de la vida situaba al ser humano en lo más alto de la copa del árbol, como la especie más evolucionada y avanzada. Los robots y la inteligencia artificial están redefiniendo el orden natural y su estructura jerárquica. No es raro, pues el orden natural siempre fluye, moldeado por los nuevos descubrimientos científicos, en especial la lectura del código genético, que revela y redefine las relaciones entre las formas de vida. Sin embargo, por primera vez, se está introduciendo una nueva especie, artificial, en los esquemas existentes y, por primera vez, parece hibridar el orden mundial natural antropocéntrico.

\section{De las tecno-especies a las tecno-personas}

EN LOS ALBORES DEL SIGLO XXI resulta evidente que, a medida que la tecnología robótica se vuelva más ubicua, el alcance de las interacciones entre robots y humanos no solo crecerá, también influirá drásticamente en las concepciones ordinarias acerca de la agencia moral. Tendencias antropomorfas en el diseño de los robots avivan nuestra percepción de la tecnología robótica como si esta estuviera inmersa en situa- 
ciones morales. En un primer momento, nos podría parecer evidente, por ejemplo, que la profesión de enfermero/a responde a cierta concepción de la responsabilidad moral, pero no nos resultaría tan claro sin embargo que dicha responsabilidad esté presente en el caso de que el cuidador sea un robot. Al mismo tiempo, la robótica social nos confronta con dilemas éticos inequívocos relacionados por ejemplo con procesos de automatización de las tecnologías de asistencia y, en general, con el aprendizaje automático, i.e. estadística computacional.

La aparición de la clase de dilemas ético-jurídicos a los que nos vamos a referir a continuación debería ser previsible para economistas e historiadores de la economía como Carlota Pérez (2002) que han defendido el argumento basado en evidencias según el cual las burbujas de inversión especulativa son una condición necesaria para el despliegue masivo de tecnologías disruptivas. Sin embargo, a pesar de dichas advertencias, los dilemas ético-jurídicos asociados a dichas etapas disruptivas no están tematizados o teorizados suficientemente debido a un déficit en la conceptualización filosófica de los mismos. Sectores como la salud, el transporte, la energía, la seguridad, la educación, etc., experimentarán transformaciones drásticas debido a la revolución real propiciada por modelos muy poderosos de computación. Una computación cuya inteligencia describimos como artificial precisamente debido a la índole de la disponibilidad de conjuntos de datos masivos que produce. No obstante, ni el desafío geopolítico ni los dilemas ético-jurídicos que se desprende de todo ello radica en la naturaleza de la materia prima de la inteligencia artificial, i.e. datos. Tampoco se aprecia un desafío radicalmente nuevo en el hecho de que grandes empresas multinacionales controlen gobiernos nacionales o el multi-gobierno europeo. No podría calificarse de innovación disruptiva el despliegue de la estadística computacional cuando esta conduce a un modelo de carrera armamentística poblada de escenarios de guerra cibernética, propaganda computacional masiva, robots asesinos, vigilancia y reconocimiento facial masivos, etc. Podemos encontrar ejemplos de tecnologías análogas en la historia económica, política y militar, y la diferencia podría establecerse únicamente desde el punto de vista de su grado o graduación (e.g. la naturaleza invasiva, letal, eficaz, propagandística y/o intrusiva de cada una de las acciones y estrategias implicadas y analizadas en cada momento).

De manera más acertada, algunos especialistas han situado la reflexión ético-jurídica en el análisis de la influencia que tendrá la planificación algorítmica de dominios cada vez más amplios y más sofisticados de la vida humana y no humana. Suele citarse a este respecto la conocida sentencia de Cathy O'Neil (2016) de acuerdo con la cual los algoritmos pueden entenderse como si se tratara de opiniones incrustadas en el código. De esta aseveración se infiere que las opiniones y los puntos de vista dominantes someterán aún más — no necesariamente de otra forma- a grupos 
minoritarios (o incluso mayorías potenciales) cuyas opiniones y puntos de vista no permeen o penetren ese código y, por consiguiente, no estén, por así decir, en línea. De ello se deriva a su vez que las sociedades humanas estarían experimentando un desafío tecnológico con un claro impacto sociopolítico y ético-jurídico pues la automatización de las decisiones (dominantes) estaría saboteando la aplicación práctica y normativa de concepciones de la imparcialidad arraigadas en la cultura jurídica de buena parte de la población mundial.

Para poder apreciar en toda su crudeza lo disruptivo de un escenario político que amenaza la aplicación de cualquier concepción de la imparcialidad es necesario introducir conceptos y concepciones de la tecnociencia que capturen los cambios experimentados y localicen fronteras y límites normativos. El filósofo Javier Echeverría ha realizado dicha investigación teórica (1999, 2002, 2003, 2013, 2014, 2017). Apoyándose en desarrollos teóricos propios anteriores, el filósofo sostiene que la noción de tecnopersona es un tema de reflexión por sí mismo. Las tecnociencias del siglo xx no solo han transformado entornos sino a las personas mismas. Esto sucede así desde el momento en que comenzaron a tecnologizarse manifestaciones y modalidades de cognición y comunicación que posteriormente incluyeron tecnologías de digitalización del devenir (e.g. acontecimientos, movimientos, impresiones sensoriales, etc. $)^{3}$. Como resultado de ello, el sistema tecnológico TIC (Tecnologías de la Información y la Comunicación) no solo digitaliza el devenir, sino que transforma al tiempo en cuanto tal (pasado y presente); y, por ello, ha dado lugar no solo a la digitalización del tiempo sino a la aparición del tecnotiempo. Esta última modalidad se caracteriza por dotar a todos los fenómenos y hechos (potencialmente) discursivos de un carácter iterativo, es decir, el tecnotiempo asegura su permanente transmisibilidad digital, su reproducción en un nuevo espacio-tiempo social que su autor denomina el tercer entorno que superpone al entorno de la physis y de la pólis. Todo esto es lo que está en juego cuando en el lenguaje ordinario se

\footnotetext{
${ }^{3}$ De acuerdo con Echeverría, la investigación doctoral de Shannon y su posterior artículo A Mathematical Theory of Communication (1948) puso de manifiesto que los circuitos eléctricos se podían analizar con ayuda del lenguaje de la lógica, de donde se infería la existencia de un isomorfismo entre las álgebras de circuitos y la lógica proposicional. Echeverría sostiene que este descubrimiento fue fundamental para la posterior aparición de lo que denomina el «sistema tecnológico TIC». Los desarrollos de las nuevas formas de lógos matemático (e.g. cálculo diferencial e integral de Leibniz y el álgebra cartesiana) son la base de aplicaciones posteriores. Según el autor, una de las innovaciones más disruptivas es el tecno-lógos, pues supone la integración en un sistema binario de las distintas lenguas y lenguajes de la ciencia, con sus respectivos signos y modalidades de representación, desde los signos a las fórmulas pasando por los gráficos y las visualizaciones. Esta innovación se ha producido en una dimensión constitutiva para la humanidad: la semiosis. El filósofo sostiene la tesis de que «la digitalización aporta un nuevo lógos tecnológico que, en primera instancia, expresa las palabras, las frases y los discursos comunes mediante códigos y expresiones digitales. Vale para todas las lenguas previamente existentes, sin excepción. Por esta razón puede ser considerada como un paso importante en la construcción de la Lingua Universalis que imaginó el joven Leibniz (...)» (Echeverría, 2017, 92).
} 
constata que la vida se halla digitalizada en las redes sociales. Echeverría propone esta terminología con el fin de sostener la hipótesis general de que el techno-lógos y las tecnopersonas están a la base del tecno-poder. Una hipótesis que si traemos aquí a colación es porque está relacionada con otra secundaria o derivada que consideremos más relevante para el tema que nos ocupa, a saber: el tecnotiempo (e.g. la digitalización) afecta a la praxis humana, a las acciones, y no solo a los objetos, a las cosas: las acciones habrían quedado absorbidas en un lógos global. Estas hipótesis están relacionadas con dos grandes conjuntos de procesos o evidencias que el autor denomina «vías»:

a) la implementación tecnológica de personas existentes (e.g. prótesis, desarrollo de artefactos para controlar capacidades perceptivas, informacionales, comunicacionales, cognitivas);

b) la dotación a máquinas y robots de capacidades similares a las de los seres humanos, con el propósito ulterior de intervenir en ellas.

Es a partir de estos conceptos y de la concepción filosófica del sistema tecnológico TIC desarrollada por este autor como cabe aproximarse al tipo de dilemas ético-jurídicos que han irrumpido en nuestra época. ¿Por qué? Porque las tecnopersonas piensan, sienten y se comunican en una especie de nuevo mundo, el tecnomundo, en el que (los archivos de) la memoria y la identidad se ubican en Big Data y repositorios digitales. Porque las tecnopersonas no tienen identidad jurídica ni se definen por contornos físicos delimitados, antes bien son únicamente el resultado de la asignación de signos de identificación atribuidos por las TIC. Porque a una sola persona cabe atribuir devenires de multitud de tecnopersonas cuyo eterno carácter iterativo dejará rastros registrados en el tercer entorno que sobrevivirán a la persona física cuya identidad jurídica y defunción solo asigna y registra el Estado. A partir de esta argumentación coordinada es como cabe entender los posibles elementos disruptivos que no hemos querido identificar sin más con la vigilancia masiva ni el fenómeno del reconocimiento facial masiva, la guerra cibernética, etc. La naturaleza de los nuevos dilemas ético-jurídicos solo se atisba si tenemos en cuenta que las tecnopersonas no tienen conciencia ni memoria ni identidad, y sin embargo se utilizan como un medio para programar y ejercer modelos de manipulación desconocidos hasta ahora ya que solo en nuestra época las personas se pueden convertir en tecnopersonas. Ahora se entenderá que cuando numerosos especialistas se preguntan cuándo un robot es un (verdadero) agente moral en realidad están aludiendo a uno de los grandes desafíos conceptuales de la filosofía política. Si tenemos 
presente lo desarrollado hasta aquí, es decir, si asumimos la vigencia de conceptos y concepciones echeverrianas, podríamos clasificar dichos desafíos para las ciencias morales y jurídicas a partir de las dos vías aludidas:

a) desafíos sociopolíticos y ético-jurídicos derivados de la implementación tecnológica de las capacidades comunicacionales de personas existentes hasta producir tecnopersonas;

b) desafíos sociopolíticos y ético-jurídicos ocasionados por la acción de dotar a máquinas y robots de capacidades en algún sentido similares a las de los seres humanos.

Contamos con un amplio repertorio de narrativas acerca de seres singulares y mundos de ciencia ficción en los que historias de tecnopersonas y robots sociales nos hacen sentir la proximidad de un futuro que comprendemos mucho mejor que nuestro propio presente. El dolor que ocasiona la muerte de un ser amado nos ayuda a empatizar con personajes que fantasean con la hipótesis de una iteración constante de registros digitales asociados a la persona fallecida en la serie de televisión británica Black Mirror. La reproducción de archivos electrónicos no solo configura el perfil de una tecnopersona, también tiene el poder de suministrar un repertorio narrativo verosímil con el que las personas que conocieron a su autor original no solo se entregan a una experiencia estética que parece alargar la vida de quien ha muerto, sino que reviven historias composibles rescatadas y recreadas a partir de fragmentos de una vida pasada y, por ello, lógicamente futurible. Tal vez tendemos a pensar que las emociones básicas que suscitan tramas narrativas inspiradas en la atribución a robots de capacidades prototípicamente humanas no pueden ir más allá del miedo o el terror. Y tal vez con el fin de contradecir esa tendencia, en la literatura científica sobre agentes morales e ingeniería de seguridad suele aludirse a la idea de que el robot puede ser «el mejor amigo del soldado». Un coronel del ejército de Estados Unidos estaba probando un robot diseñado para desactivar minas terrestres al pisarlas. Cada vez que pisaba una mina el robot perdía una de sus piernas. Durante la operación, el coronel no pudo soportar ver cómo se arrastraba el robot que se quemaba y desgarraba mientras cumplía su misión: el agente autónomo consiguió evocar (presuntos) valores morales como la lealtad, la abnegación o el autosacrificio.

Sin embargo, a pesar del impacto y la claridad (estética) con la que experimentamos controversias y dilemas incrustados en tramas narrativas visuales y literarias inspiradas en alguna de las dos vías referidas, i.e. tecnopersonas y robots sociales, 
lo cierto es que su dispersión teórica es manifiesta cuando elevamos su nivel de formulación teórica y leemos textos mucho más sofisticados desde el punto de vista normativo. Este es el caso, por ejemplo, de una polémica resolución aprobaba por el Parlamento Europeo.

En 2017 el Parlamento Europeo aprobaba una resolución con recomendaciones destinadas a la Comisión acerca de las normas de Derecho civil sobre robótica. Todos sabemos que este tipo de documentos se redactan con diligencia, eliminando toda clase de licencia literaria y con la finalidad de ser inequívoco e incluso expeditivo. Sin embargo, en este caso, por lo que se ve, los parlamentarios sucumbieron al poderoso efecto de la producción literaria a cuyos fantásticos repertorios fueron incapaces de sustraerse mientras cumplían su función: ser eficaces comunicativamente e incluso objetivos. Y acordaron escribir sin voluntad alguna de estilo literario lo que sigue: las primeras líneas de una posible novela o un tratado filosófico futurista:

Considerando que, desde el monstruo de Frankenstein creado por Mary Shelley al mito clásico de Pigmalión, pasando por el Golem de Praga o el robot de Karel Čapek — que fue quien acunó el término- - los seres humanos han fantaseado siempre con la posibilidad de construir máquinas inteligentes, sobre todo androides con características humanas (...) [el Parlamento] pide a la Comisión que proponga definiciones europeas comunes de sistema ciberfísico, sistema autónomo, robot autónomo inteligente y sus distintas subcategorías (...). Resolución del Parlamento Europeo, del 16 de febrero de 2017 (Introducción).

Tan solo unos meses después, más de doscientos expertos europeos dirigieron una carta abierta al presidente de la Comisión Europea, Jean-Claude Juncker, para mostrar su desacuerdo con la idea de conceder personalidad (electrónica) a los robots. La idea de dotar de personalidad jurídica a las máquinas y, por tanto, la atribución a los robots de la capacidad y responsabilidad de reparar a alguien del daño ocasionado no dejó indiferentes a 285 europarlamentarios que votaron en contra del párrafo más controvertido de la resolución, el número 59 (f). Ante la dificultad de dirimir bajo qué condiciones y circunstancias los daños que pudiera ocasionar un robot son imputables al fabricante, a la empresa que provee el software o, por el contrario, al usuario, y como estas dificultades suelen representar un claro percance para las personas afectadas que solo desean ser indemnizadas, las compañías aseguradoras ejercieron presiones sobre los europarlamentarios para resolver el dilema a través de un atajo propio de la ciencia ficción: otorgar personalidad electrónica a todo robot facilitaría la posterior atribución de responsabilidad civil a los mismos ${ }^{4}$.

\footnotetext{
${ }^{4}$ En la sección en la que se enumeran los Principios generales relativos al desarrollo de la robótica y la inteligencia artificial para uso civil se puede leer: «59 f) crear a largo plazo una personalidad jurídica específica para los robots, de forma que como mínimo los robots autónomos más complejos puedan ser considerados personas electrónicas
} 
Las tecnopersonas no forman parte siquiera de esta controversia jurídica, ellas no han suscitado un debate equiparable: los registros y rastros electrónicos que adornan y sustancian nuestra huella digital son simples despojos, equiparables a desechables. Ni siquiera tenemos la obligación de identificarnos con dichos registros o reclamar su autoría, aunque nos sobrevivan. Cabe suponer que los recorridos normativos asociados a cada singularidad (tecnopersonas, robots) serán muy diferentes. La controversia en torno a la presunta agencia moral de los robots no es en absoluto equiparable al debate acerca de la autonomía que pueda atribuirse a las tecnopersonas. Y ello se debe no solo a diferencias relativas a la apropiación semántica de los términos sino al hecho de que en el entorno digital los poderes mediáticos, i.e. el tecnopoder, se configuran a través de una computación ubicua, cuyas acciones no adquieren contornos claramente antropomorfos como los que, en contraposición, todos creemos atisbar en los robots diseñados para prestar servicios de cuidado los cuales, en algunos contextos culturales, llegan a relacionarse incluso con concepciones animistas, tal y como se desprende de la investigación de Kovacic (2018).

De lo anterior se infiere que los desafíos normativos relacionados con las tecnopersonas y los robots no se pueden plantear sin atender antes a intersecciones de la tecnociencia con valores morales presentes en la cognición social de los grupos humanos. Pongamos el ejemplo de la conducción automatizada de vehículos. Una de sus aparentes ventajas es que sabemos que los autómatas no utilizan parámetros mentalistas —en el sentido de que no aplican ninguna concepción de teoría de la mente ni necesitan leer o adivinar estados mentales - para efectuar cálculos y proceder a su ulterior ejecución en la forma de decisiones automáticas. La clave de ello radica en la razón a la que alude Fernández Castro (2017) en un interesante trabajo: los cálculos de los robots sencillamente están basados en las normas de tráfico. Ahora bien, esta explicación en realidad resulta más compleja si asumimos que la cognición social no constituye un dominio independiente (lógicamente) del entorno cultural. Nos podríamos preguntar por ejemplo cómo afectaría la tendencia a asumir posiciones animistas - mucho más frecuente comparativamente en Japón que en cualquier país europeo- a la hora de analizar cómo perciben los seres humanos la robótica aplicada a la automoción. Siempre habrá conductores que presenten una clara tendencia a calcular el comportamiento de otros conductores (incluso, y sobre todo, si se trata de robots) asumiendo parámetros propios de teoría de

responsables de reparar daños que puedan causar, y posiblemente aplicar la personalidad electrónica a aquellos supuestos en los que los robots tomen decisiones autónomas inteligentes o interactúen con terceros de forma independiente.» Resolución del Parlamento Europeo, del 16 de febrero de 2017, con recomendaciones destinadas a la Comisión sobre Normas de Derecho Civil sobre Robótica (2015/2013(INL)). 
la mente. No parece tan claro por consiguiente que los sistemas robóticos puedan eludir la compleja intersección entre ciencia, tecnología y valores si asumimos que los robots sociales —al igual que los seres humanos- están diseñados precisamente para involucrarse en la interacción social. 


\section{REFERENCIAS BIBLIOGRÁFICAS}

Braidotti, Rosi, "Posthuman, All too Human: Towards a New Process Ontology." Theory, Culture \& Society. Ed. Nicholas Gane. Sage, 2006. 197-208. DOI: https://doi.org/10.1177/0263276406069232

ČApeK, Karel, R.U.R. Mineola, NY, Dover Publications Inc., 2001.

Clyne, Manfred E. and Kline, Nathan S., "Cyborgs and Space." Astronautics 5(9), 26-27, 74-76, 1960.

Darwin, Charles, The Origins of Species by Means of Natural Selection or the Preservation of Favoured Races in the Struggle for Life, London, ElecBook. 1997. DOI: https://doi.org/10.5962/bhl.title.82303

Descartes, René, Treatise of Man, Cambridge, Mass, Harvard University Press, 1972. DOI: https://doi.org/10.1017/CBO9780511605727.008

ECheVerría, Javier, Los señores del aire, Telépolis y el tercer entorno, Barcelona, Destino, 1999. DOI: http://dx.doi.org/10.20350/digitalCSIC/9185

---, Ciencia y valores, Barcelona, Ediciones Destino, 2002.

---, La revolución tecnocientifica, Madrid, FCE, 2013.

---, Innovation and Values. A European Perspective, Reno, NV, UNR/CBS, 2014.

---, “Tecnociencias e innovaciones: desafíos filosóficos. Las tecnopersonas como ejemplos." Actas II Congreso Internacional de la Red Española de Filosofía VIII 2017, 89-103.

---, El arte de innovar. Naturalezas, lenguajes, sociedades, Madrid, Plaza y Valdés, 2017.

Fernández Castro, Víctor, "Mindshaping and Robotics." Sociality and Normativity for Robots: Philosophical Inquiries into Human-Robots Interactions. Ed. Raul Hakli y Johanna Seibt. Springer, 2017, 115-135. DOI: https://doi. org/10.1007/978-3-319-53133-5_6

Gehlen, Arnold, Man: His Nature and Place in the World, New York, Columbia University Press, 1988.

Haraway, Donna, "A Cyborg Manifesto: Science, Technology, and Socialist-Feminism in the late Twentieth Century." Simians, Cyborgs, and Women: The Reinvention of Nature. Ed. Donna Haraway. London: Routledge, 1991, 149-181. DOI: https://doi.org/10.1007/978-1-4020-3803-7_4 
Hayles, Katherine, "Flesh and Metal: Reconfiguring the Mindbody in Virtual Environments." Data Made Flesh: Embodying Information. Eds. Robert Mitchell and Phillip Thurtle. New York: Routledge, 2004. DOI: doi:10.1353/ con.2003.0015.

---, "Unfinished Work: From Cyborg to Cognisphere." Theory, Culture \& Society. Thousand Oaks: Sage, 2006, 159-166. DOI: https://doi. org/10.1177/0263276406069229

Koestler, Arthur, The Ghost in the Machine, London, Hutchinson, 1967. DOI: DOI: 10.1126/science.160.3828.649

Kovacic, Mateja, Technologies and Paradigms of Vision: From the Scientific Revolution of the Edo Period to Contemporary Japanese Animation, Hong Kong Baptist University, Hong Kong, 2016. https://repository.hkbu.edu.hk/etd_oa/31

---, "The Making of National Robot History in Japan: Monozukuri, Enculturation and Cultural Lineage of Robots." Critical Asian Studies 50(4), 2018. DOI: https://doi.org/10.1080/14672715.2018.1512003

Laurent, Béatrice, "Monster or Missing Link? The Mermaid and the Victorian Imagination." Cahiers victoriens et édouardiens 87, 2018. DOI: https://doi. org/10.4000/cve.3188

Menzel, Peter y D’Aluisio, Faith, Robo Sapiens: Evolution of a New Species, Cambirdge, Mass, The MIT Press, 2000. DOI: https://doi.org/10.1017/ S0263574700233213

MEXT (Ministry of Education, Culture, Sports, Science and Technology). White Paper on Science and Technology. 2016/

O'Neil, Cathy, Weapons of Math Destruction: How Big Data Increases Inequality and Threatens Democracy, New York, Crown, 2016. DOI: https://doi. org/10.1177/0256090919853933

Pérez, Carlota, Technological Revolutions and Financial Capital: The Dynamics of Bubbles and Golden Ages, Cheltenham, UK, Edward Elgar, 2002. DOI: https://doi.org/10.1017/S002205070348193X

Resolución del Parlamento Europeo, del 16 de febrero de 2017, con recomendaciones destinadas a la Comisión sobre Normas de Derecho Civil sobre Robótica (2015/2013(INL)): http://www.europarl.europa.eu/sides/getDoc.do?pubRef=-//EP//NONSGML+TA+P8-TA-2017-0051+0+DOC+PDF+V0//ES 
Robertson, Jennifer, Robo Sapiens Japanicus: Robots, Gender, Family, and the Japanese Nation, Oakland: University of California Press, 2018. DOI: https:// doi.org/10.1093/ssjj/jyz002

Shannon, Claude E., "A Mathematical Theory of Communication." The Bell System Technical Journal 27, 1948, 379-423. DOI: 10.1002/j.1538-7305.1948. tb01338.x

Shelley, Mary, Frankenstein, New York, Open Road Media, 2014.

Tatsumi, Takayuki, Full Metal Apache: Transactions between Cyberpunk Japan and Avant-pop America, Durham and London, Duke University Press, 2006. DOI: https://doi.org/10.1215/9780822388012

The Computing Community Consortium Workshop. A Roadmap for U.S. Robotics: From Internet to Robotics, Chapter 3, 2009, 51-71.

Tomas, David, "Feedback and Cybernetics: Reimaging the Body in the Age of Cybernetics." Cyberspace, Cyberbodies, Cyberpunk: Cultures of Technological Embodiment. Eds. Mike Featherstone and Roger Burrows, London, Sage, 1995, 21-44. DOI: http://dx.doi.org/10.4135/9781446250198.n2

Wiener, Norbert, Cybernetics: Or Control and Communication in the Animal and the Machine, New York and Paris, The Technology Press, John Wiley and Sons Inc., Hermann et CIE, 1948.

DOI: https://doi.org/10.15366/bp2021.27.002

Bajo Palabra. II Época. No 27. Pgs: 45-62 